\title{
A Turnover in the Radio Light Curve of GW170817
}

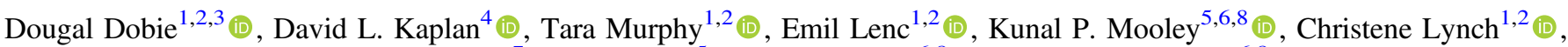

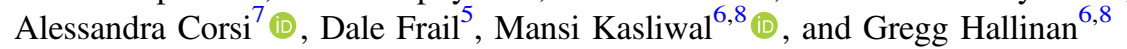 \\ ${ }^{1}$ Sydney Institute for Astronomy, School of Physics, University of Sydney, Sydney, New South Wales 2006, Australia; tara.murphy@sydney.edu.au \\ ${ }^{2}$ ARC Centre of Excellence for All-sky Astrophysics (CAASTRO), Australia \\ ${ }^{3}$ ATNF, CSIRO Astronomy and Space Science, P.O. Box 76, Epping, New South Wales 1710, Australia \\ ${ }^{4}$ Department of Physics, University of Wisconsin-Milwaukee, Milwaukee, WI 53201, USA \\ ${ }^{5}$ National Radio Astronomy Observatory, Socorro, NM 87801, USA \\ ${ }^{6}$ Caltech, 1200 East California Boulevard, MC 249-17, Pasadena, CA 91125, USA \\ ${ }^{7}$ Department of Physics and Astronomy, Texas Tech University, Box 41051, Lubbock, TX 79409-1051, USA \\ Received 2018 March 18; revised 2018 April 26; accepted 2018 April 27; published 2018 May 9
}

\begin{abstract}
We present 2-9 GHz radio observations of GW170817 covering the period 125-200 days post-merger, taken with the Australia Telescope Compact Array (ATCA) and the Karl G. Jansky Very Large Array (VLA). Our observations demonstrate that the radio afterglow peaked at $149 \pm 2$ days post-merger and is now declining in flux density. We see no evidence for evolution in the radio-only spectral index, which remains consistent with optically thin synchrotron emission connecting the radio, optical, and X-ray regimes. The peak implies a total energy in the synchrotron-emitting component of a few $\times 10^{50} \mathrm{erg}$. The temporal decay rate is most consistent with mildly or non-relativistic material and we do not see evidence for a very energetic off-axis jet, but we cannot distinguish between a lower-energy jet and more isotropic emission.
\end{abstract}

Key words: gravitational waves - radio continuum: stars - stars: neutron

\section{Introduction}

The neutron star merger GW170817 was detected via the concurrent observation of gravitational waves (Abbott et al. 2017a) and a $\gamma$-ray burst (GRB; Abbott et al. 2017b; Goldstein et al. 2017). The merger was localized to its host galaxy, NGC 4993, by the detection of an optical transient (Abbott et al. 2017c; Arcavi et al. 2017; Coulter et al. 2017; Lipunov et al. 2017; Soares-Santos et al. 2017; Tanvir et al. 2017; Valenti et al. 2017) and subsequent ultraviolet, optical, and infrared observations found evidence of kilonova emission from the source (Arcavi et al. 2017; Cowperthwaite et al. 2017; Drout et al. 2017; Evans et al. 2017; Kasliwal et al. 2017). X-ray observations found no evidence of emission until nine days post-merger (Haggard et al. 2017; Evans et al. 2017; Margutti et al. 2017; Troja et al. 2017), suggesting that this event differs significantly from previously observed GRBs.

Radio emission from GW170817 was first detected 16 days post-merger (Hallinan et al. 2017). Follow-up observations over the next 100 days (Alexander et al. 2017; Margutti et al. 2018; Mooley et al. 2018; Troja et al. 2018) revealed a gradually rising light curve. The observed radio emission follows a power law with temporal index $\delta=0.78 \pm 0.05$ and spectral index $\alpha=-0.61 \pm 0.05$, where $S_{\nu}(t, \nu) \propto t^{\delta} \nu^{\alpha}$ (Mooley et al. 2018). The observed radio spectral energy distribution agrees with the spectral index connecting contemporaneous radio, optical, and X-ray measurements, implying a common source for the observed synchrotron emission (Levan et al. 2017; Mooley \& Mooley 2017; Margutti et al. 2018; Mooley et al. 2018; Troja \& Piro 2018).

The late turn-on of the X-ray and radio emission from GW170817 is not consistent with emission produced via an onaxis relativistic jet (Alexander et al. 2017; Haggard et al. 2017;

\footnotetext{
${ }^{8}$ Jansky Fellow (NRAO/Caltech).
}

Hallinan et al. 2017; Margutti et al. 2017; Troja et al. 2017). Moreover, the gradual rise of the radio light curve rules out prompt $\gamma$-ray emission originating from a jet with a "top-hat" azimuthal density profile observed off-axis, which would have produced a much steeper peak and decline than observed (Granot et al. 2002; Nakar et al. 2002). Instead, the light curve is consistent with mildly relativistic quasi-spherical outflow called a "cocoon" (Hallinan et al. 2017; Gottlieb et al. 2018; Mooley et al. 2018; Nakar \& Piran 2018), which may have some contribution from an embedded relativistic jet observed off-axis (some versions of which are also referred to as a "structured jet"; Lazzati et al. 2017; D'Avanzo et al. 2018; Margutti et al. 2018; Resmi et al. 2018).

Based on the data available in the literature to date, it is not possible to establish whether or not a successful jet is present within the cocoon, as these scenarios exhibit similar behavior in the early stages of the afterglow evolution, or to determine the energy of the cocoon itself (see Figure 5 of Margutti et al. 2018). The timescale of the peak flux density and the rate of decline afterward can constrain the total energy of the outflow and the properties of a successful jet (if present). If the jet did not successfully break out of the cocoon (a choked jet) the observed emission is dominated by the quasi-spherical outflow (cocoon or dynamical ejecta; Gottlieb et al. 2018) and the light curve will continue to rise; if the jet is successful (a structured jet; Margutti et al. 2018; Nakar \& Piran 2018) the light curve peaks sooner and declines more rapidly. In either case, identifying when and how the light curve peaks also allows calorimetry of the cocoon emission (much as was done by Frail et al. 2000; Berger et al. 2004 for long GRBs).

To date, X-ray observations provide conflicting evidence as to whether the afterglow has peaked. XMM-Newton observations 135 days post-merger suggest the afterglow may have flattened (D'Avanzo et al. 2018), but Chandra observations show a continued rise or slow turnover at about 150 days postmerger (Haggard et al. 2018; Margutti et al. 2018; Troja \& Piro 


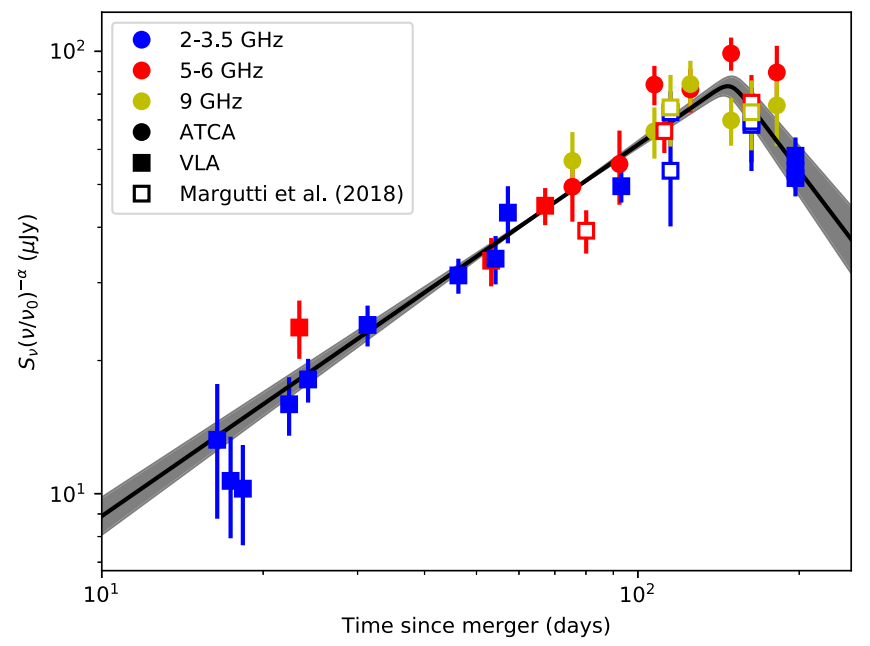

Figure 1. Light curve of GW170817 from ATCA (circles) and VLA (squares) observations grouped by frequency band, with $2-3.5 \mathrm{GHz}$ (blue), $5-6 \mathrm{GHz}$ (red), and $9 \mathrm{GHz}$ (yellow). The flux densities have been adjusted to $5.5 \mathrm{GHz}$ assuming a spectral index of $\alpha=-0.57 \pm 0.04$ (Section 3.1). Open squares denote observations from Margutti et al. (2018), while filled symbols denote observations from this Letter or other observations by our group (Hallinan et al. 2017; Mooley et al. 2018). Our best-fit smoothed broken power law with temporal index on the rise $\delta_{1}=0.84 \pm 0.05$, temporal index on the decay $\delta_{2}=-1.6 \pm 0.2$, and peak time $t_{\text {peak }}=149 \pm 2$ days is shown in black, with uncertainties shaded.

2018). A decreasing X-ray brightness would imply that either the synchrotron cooling frequency has shifted below the X-ray band (expected on timescales of 100-1000 days post-merger) and the spectrum of the source has evolved, or the light curve of the source from the radio to X-rays has peaked, but current data are not definitive that any change in the X-ray light curve has occurred.

We present further radio observations of GW170817 using the the Australia Telescope Compact Array (ATCA) and the Karl G. Jansky Very Large Array (VLA), covering the period 125-200 days post-merger. These observations demonstrate (Figure 1) that the radio afterglow has peaked at $149 \pm 2$ days post-merger and is now declining in flux density.

\section{Observations and Data Reduction}

\subsection{ATCA}

We observed GW170817 on 2017 December 20 and 2018 January 13 UT with the ATCA (PI: T. Murphy). Further observations of GW170817 with the ATCA were obtained on 2018 February 01 and 15 UT (PI: E. Troja); see Table 1 for details. The February 01 observation only had four out of six antennas available and after removing short baselines due to the compact configuration, the data quality was insufficient to make a meaningful measurement and the observation was discarded. We determined the flux scale and bandpass response for all epochs using the ATCA primary calibrator PKS B1934 -638. Observations of PKS B1245-197 were used to calibrate the complex gains during the December and January observing epochs, while PKS B1244-255 was used in the February observation. All observations used two bands of $2048 \mathrm{MHz}$ centered at 5.5 and $9.0 \mathrm{GHz}$.

We reduced the visibility data using standard MIRIAD (Sault et al. 1995) routines. The calibrated visibility data were split into the 5.5 and $9.0 \mathrm{GHz}$ bands, averaged to $32 \mathrm{MHz}$ channels, and imported into DIFMAP (Shepherd 1997). Bright field sources were modeled separately for each band using the visibility data and a combination of point-source and Gaussian components with power-law spectra. After subtracting the modeled field sources from the visibility data, GW170817 dominates the residual image. Restored naturally weighted images for each band were generated by convolving the restoring beam and modeled components, adding the residual map and averaging to form a wideband image. Image-based Gaussian fitting with unconstrained flux density and source position was performed in the region near GW170817. The resulting source position agrees with the position of GW170817 observed by the Hubble Space Telescope (HST; Adams et al. 2017).

To examine the stability of the absolute flux calibration from epoch to epoch we measured the flux density of the phase calibrator (PKS B1245-197) and a compact reference source in the GW170817 field (R.A. $=13^{\mathrm{h}} 09^{\mathrm{m}} 53.91$, decl. $=-23^{\circ} 21^{\prime}$ 34. . 5, 1!.9 from GW170817) in each epoch and frequency band of the ATCA data. We do not use the host galaxy NGC 4993 as it is extended. We find that the mean and standard deviation of the phase calibrator flux density is $2.193 \pm 0.013 \mathrm{Jy}$ and $1.449 \pm 0.021 \mathrm{Jy}$ at $5.5 \mathrm{GHz}$ and $9 \mathrm{GHz}$, respectively. This compares to within $0.1 \%$ with the values reported by the ATNF Calibrator Database. ${ }^{9}$ The reference source is three orders of magnitude fainter than the phase calibrator but is a factor of at least three brighter than GW170817 and is within the same field, so it should provide an accurate indication of the flux density scale within the target field itself. The source is also visible regardless of which phase calibrator is used and so provides an independent test of flux scale stability. Across all epochs, we find that the mean flux density and standard deviation of the reference source flux density is $452 \pm 16 \mu \mathrm{Jy}$ and $301 \pm 18 \mu \mathrm{Jy}$ at $5.5 \mathrm{GHz}$, and $9.0 \mathrm{GHz}$, respectively. This suggests that our field flux density measurements are stable to within $2.9 \%$ and $5.4 \%$ at $5.5 \mathrm{GHz}$ and $9.0 \mathrm{GHz}$, respectively, where those additional uncertainties when added in quadrature to the measurement uncertainties give reduced $\chi^{2}=1$ for the reference source. For GW170817 itself we measured the noise in the vicinity of the source to account for additional contributions from unmodeled sidelobes from the host galaxy NGC 4993 and included the additional uncertainties discussed above.

\subsection{VLA}

VLA observations of the GW170817 field were carried out on 2018 March 02 (Table 1). The Wideband Interferometric Digital Architecture correlator was used at $\mathrm{S}$ band $(2-4 \mathrm{GHz})$ to maximize sensitivity. We used J1248-1959 as the phase calibrator and 3C286 as the flux density and bandpass calibrator. The data were calibrated and flagged for radio frequency interference (RFI) using the NRAO CASA (McMullin et al. 2007) pipeline. We then split and imaged the target data using the CASA tasks split and clean. We made final images by splitting the bandpass into two subbands of $1 \mathrm{GHz}$ each.

\footnotetext{
9 http://www.narrabri.atnf.csiro.au/calibrators/
} 
Table 1

New Radio Observations of GW170817

\begin{tabular}{|c|c|c|c|c|c|c|}
\hline UT Date & $\begin{array}{c}\Delta T \\
\text { (day) }\end{array}$ & Telescope & $\begin{array}{c}\nu \\
(\mathrm{GHz})\end{array}$ & $\begin{array}{l}\text { Bandwidth } \\
\text { (GHz) }\end{array}$ & $\begin{array}{c}\text { Beam Size } \\
(\operatorname{arcsec})\end{array}$ & $\begin{array}{c}S_{\nu} \\
(\mu \mathrm{Jy})\end{array}$ \\
\hline \multirow[t]{2}{*}{2017 Dec 20.83} & 125.30 & $\mathrm{ATCA}^{\mathrm{a}}$ & 5.5 & 2.048 & $5.8 \times 1.5$ & $82.0 \pm 9.3$ \\
\hline & & & 9.0 & 2.048 & $3.6 \times 1.0$ & $63.7 \pm 8.2$ \\
\hline 2018 Jan 13.79 & & & 9.0 & 2.048 & $3.3 \times 1.0$ & $52.7 \pm 6.5$ \\
\hline \multirow[t]{2}{*}{2018 Feb 01.74} & 168.21 & $\mathrm{ATCA}^{\mathrm{b}}$ & 5.5 & 2.048 & $\ldots$ & $\ldots^{c}$ \\
\hline & & & 9.0 & 2.048 & $\ldots$ & $\ldots^{\mathrm{c}}$ \\
\hline \multirow[t]{2}{*}{2018 Mar 02.32} & 196.79 & VLA $^{\mathrm{e}}$ & 2.5 & 1 & $1.3 \times 0.5$ & $91.0 \pm 9.1$ \\
\hline & & & 3.5 & 1 & $1.3 \times 0.5$ & $66.9 \pm 6.1$ \\
\hline
\end{tabular}

Notes.

${ }^{a}$ With the 6C configuration (maximum baselines of $6 \mathrm{~km}$ ) and program CX391 (PI: T. Murphy).

${ }^{\mathrm{b}}$ With the $750 \mathrm{~A}$ configuration (maximum baseline of $3.75 \mathrm{~km}$ ) and program CX394 (PI: E. Troja).

${ }^{\mathrm{c}}$ Insufficient data quality.

${ }^{\mathrm{d}}$ With the 750B configuration (maximum baseline of $4.5 \mathrm{~km}$ ) and program CX394 (PI: E. Troja).

${ }^{\mathrm{e}}$ With the A configuration (maximum baseline of $27 \mathrm{~km}$ ) under a Director Discretionary Time program (VLA/17B-397; PI: K. Mooley).

\section{Results and Discussion}

\subsection{Spectral Analysis}

We first revisit the spectral behavior of the radio emission. As in Mooley et al. (2018) we fit a power law of the form $S_{\nu} \propto \nu^{\alpha} t^{\delta}$ to the first 120 days of the radio light curve (before any sign of a turnover) and find a spectral index $\alpha=$ $-0.57 \pm 0.04$ and temporal index $\delta=0.84 \pm 0.05$. This is consistent with Mooley et al. (2018) and with Margutti et al. (2018), who find a joint radio-to-X-ray spectral index $\alpha=$ $-0.585 \pm 0.005$ at 110 days and $\alpha=-0.584 \pm 0.006$ at 160 days post-merger.

We examined the variability of the spectral behavior using all quasi-simultaneous radio observations. We identified data sets with more than one observation within \pm 1 day and fit for a spectral index. These values are shown in Figure 2. We find the data largely consistent with a constant spectral index, with $\chi^{2}=15.9$ for 12 degrees of freedom. There appears to be no evidence for significant change in the spectrum of the source, consistent with previous radio, X-ray, and HST observations (D'Avanzo et al. 2018; Lyman et al. 2018; Margutti et al. 2018; Mooley et al. 2018; Resmi et al. 2018).

\subsection{Light Curve Analysis}

Figure 1 shows the light curve of GW170817 over the 2-9 GHz frequency range from the observations in Table 1 and the literature (Hallinan et al. 2017; Margutti et al. 2018; Mooley et al. 2018), scaling the flux density for each observation to $5.5 \mathrm{GHz}$ based on the spectral index of $\alpha=-0.57 \pm 0.04$ calculated above. Assuming the light curve initially rises with a temporal index of $\delta_{1}=0.84$, peaks $t_{\text {peak }}$ days post-merger, and fades with a temporal index of $\delta_{2}$, we fit a smoothed broken power law ${ }^{10}$ using the Astropy modeling package (The Astropy Collaboration et al. 2018) that behaves as $S_{\nu} \propto t^{\delta_{1}}$ for $t \lesssim t_{\text {peak }}$ and $S_{\nu} \propto t^{\delta_{2}}$ for $t \gtrsim t_{\text {peak }}$ with a smooth transition around $t \approx t_{\text {peak }}$. We do not expect to see any variability due to interstellar scintillation, due to the source size (Hallinan et al. 2017).

\footnotetext{
$\overline{10} \mathrm{http}: / /$ docs.astropy.org/en/stable/api/astropy.modeling.powerlaws. SmoothlyBrokenPowerLaw1D.html
}

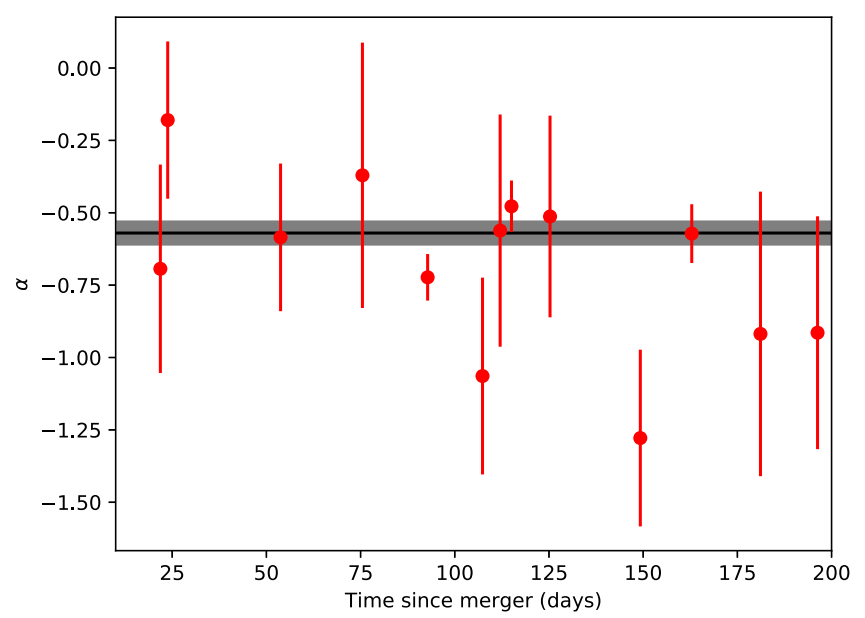

Figure 2. Spectral index $(\alpha)$ of contemporaneous radio observations from Hallinan et al. (2017), Mooley et al. (2018), Margutti et al. (2018), and this work. The best-fit spectral index from the first 120 days of the radio light curve, $\alpha=-0.57 \pm 0.04$, is shown in black, with uncertainties shaded.

We have fit the light curve allowing the smoothing factor to freely vary and find a minor preference for small smoothing factors down to 0.001 , corresponding to a transition of 0.3 days either side of the break. To approximate our observing cadence near the peak of the light curve we use a smoothing factor of 0.02 (corresponding to a $<20$ day transition), which produces no significant changes in fit parameters.

Figure 3 shows the two-dimensional joint confidence region as a function of $t_{\text {peak }}$ and $\delta_{2}$, where we indicate the best-fit values, $\delta_{2}=-1.6 \pm 0.2$ and $t_{\text {peak }}=149 \pm 2$ days, and the $90 \%$ confidence region. The best fit has $\chi^{2}=41.6$ for 35 degrees of freedom. For a radio light curve that is continuing to rise, the temporal index would remain the same, $\delta_{2}=\delta_{1}$, which we indicate with the dashed line in Figure 3. Comparing the $\chi^{2}\left(\delta_{2}=\delta_{1}\right)$ to the minimum $\chi^{2}$ for $\delta_{2}=-1.6$, we find a change of 380 for one additional parameter and can exclude a light curve that continues to rise at greater than $5 \sigma$ significance using an F-test. We further find a change of $\chi^{2}$ of 35 from $\delta_{2}=0$ to the best-fit value $\delta_{2}=-1.6 \pm 0.2$, leading to a 


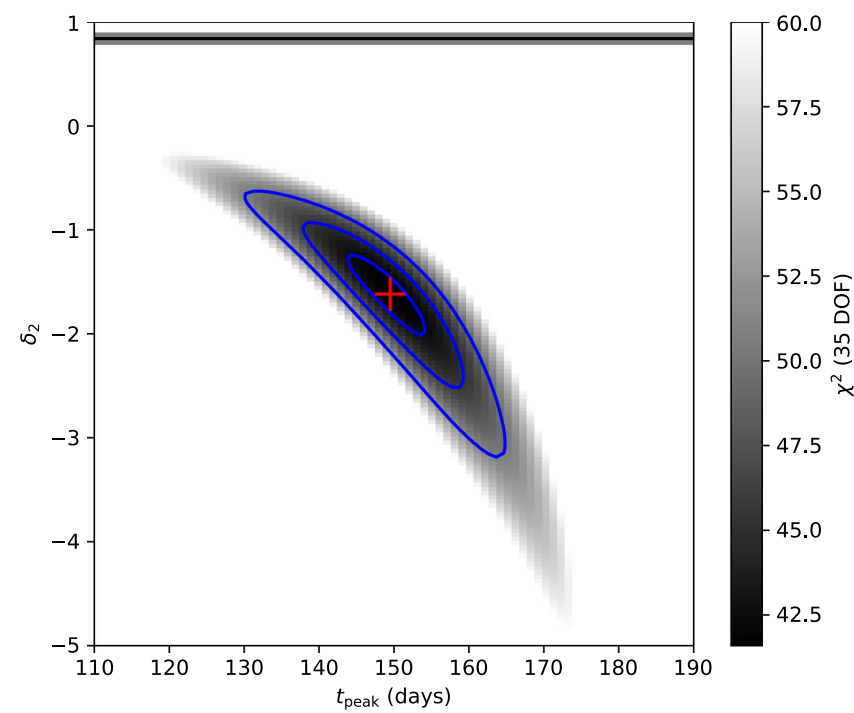

Figure 3. Two-dimensional joint probability distribution of $\delta_{2}$ and $t_{\text {peak }}$, assuming $\alpha=-0.57 \pm 0.04$ and $\delta_{1}=0.84$. The background grayscale is the $\chi^{2}$ for 35 degrees of freedom, with 1-, 2-, and 3- $\sigma$ joint confidence contours are shown in blue. The best-fit value of $\delta_{2}=-1.6 \pm 0.2$ and $t_{\text {peak }}=149 \pm$ 2 days is shown in red. The temporal index of the light curve as it rises, $\delta_{1}=0.84 \pm 0.05$, is indicated by the black line with uncertainties shaded.

declining light curve. Preliminary reduction of further observations confirms the observed trend.

\subsection{Interpreting the Radio Light Curve}

The observed light curve turns over and declines with no evidence for a steep rise coming with an energetically dominant off-axis jet (Nakar \& Piran 2018), but a weaker jet may still be present. The relatively sharp peak in the radio light curve implies that the energy injection has reduced substantially (or stopped), or that the ejecta has collected mass comparable to its own. The former scenario would be relevant for a successful jet (e.g., Kasliwal et al. 2017; Lazzati et al. 2017; D’Avanzo et al. 2018; Margutti et al. 2018; Mooley et al. 2018; Troja et al. 2018) or a low-energy choked-jet cocoon (e.g., Kasliwal et al. 2017; Gottlieb et al. 2017; Piro \& Kollmeier 2018; Mooley et al. 2018), while the latter would be relevant in the case of an isotropic fireball (i.e., dynamical ejecta; Nakar \& Piran 2011; D'Avanzo et al. 2018; Hotokezaka et al. 2018; Mooley et al. 2018).

While no substantial degree of linear polarization would be expected from isotropic dynamical ejecta, in the successful jet model the required asymmetry is built into the jet structure (the energy and speed of the various ejecta components are both functions of the angle from the jet axis; see e.g., Lazzati et al. 2017). Thus, the relevant emitting surface is never completely symmetric for misaligned observers, resulting in an appreciable degree of linear polarization ( $20 \%$; Rossi et al. 2004). A detection of significant linear polarization would thus point to a successful jet rather than isotropic dynamical ejecta (also see Gill \& Granot 2018).

The radio light curve can give the energy profile of the ejecta, but it is not sufficient for distinguishing between the contributions from radial and angular structures within the ejecta. Very Long Baseline Interferometry (VLBI) can, however, provide images at sub-milliarcsecond angular resolution, and thus constrain the geometry of the outflow.
Distinguishing between the successful-jet, choked-jet cocoon, and dynamical ejecta models is thus possible using VLBI observations.

The time of the radio peak is near the observed plateau on the X-ray light curve (D'Avanzo et al. 2018; Margutti et al. 2018; Ruan et al. 2018; Troja et al. 2018), and suggests that the $\mathrm{X}$-rays peaked at the same time as the radio light curve. The turnover in the X-ray (and radio) light curve is therefore dynamical or geometric in origin, and the cooling break has (likely) not entered the X-ray band yet. This is consistent with the interpretation of D'Avanzo et al. (2018) and Margutti et al. (2018), who found that the radio, optical, and X-rays lie on the same power law until day 150 post-merger.

The light curve of a relativistic jet afterglow will decay as $t^{-p}$, while in the non-relativistic regime the decline will be proportional to $t^{(15 p-21) / 10}$, with $p$ the exponent on the distribution of electron energies, $N(E) \propto E^{-p}$ (Granot et al. 2002; Nakar \& Piran 2011). In the case of GW170817, $p=2.17$ (e.g., Margutti et al. 2018; Mooley et al. 2018), so the expected decay slopes are $t^{-2.2}$ and $t^{-1.2}$. Our radio data are consistent with expectations for the mildly or non-relativistic regimes. Based on the time and the flux density at the peak of the radio light curve, we can further calculate the isotropicequivalent energy (Nakar \& Piran 2018) as a few $\times 10^{50}$ erg for the cocoon scenario (also see Resmi et al. 2018) and a few $\times 10^{49}$ erg for the dynamical ejecta scenario. Both of those are lower than the isotropic-equivalent kinetic energies found for short GRBs (Fong et al. 2015).

If the peak of the light curve was dominated by an off-axis jet, then $\Gamma\left(\theta_{\text {obs }}-\theta_{\text {jet }}\right) \simeq 1$ Nakar \& Piran (2018; where the bulk Lorentz factor of the jet is $\Gamma$, the off-axis angle of the observer is $\theta_{\text {obs }}$, and the opening angle of the jet is $\left.\theta_{\text {jet }}\right)$ implies that $\left(\theta_{\text {obs }}-\theta_{\text {jet }}\right) \simeq 20^{\circ}$, assuming that material with $\Gamma \simeq 3$ dominated the on-axis emission at peak. Therefore, we can constrain $\theta_{\text {jet }} \lesssim 8^{\circ}$ using the viewing angle constraint from the Laser Interferometer Gravitational-Wave Observatory (LIGO)/ Virgo $\left(\theta_{\text {obs }}<28^{\circ}\right.$; Abbott et al. 2017a).

Continued radio monitoring will be essential for constraining the decay index. A steep decline in the radio light curve would favor the scenario in which a successful jet broke out of the dynamical ejecta. Transition of the ejecta from the mildly relativistic to the Newtonian regime would be characterized by deviation from a power-law decay and a change in spectral index, which could be detected with sensitive follow-up observations. It is even possible for the ejecta to have angular structures that could cause the light curve to rise again: the early-time kilonova signal in the optical suggested the presence of $\sim 0.05 M_{\odot}$ material traveling at speeds of $0.1 c$ to $0.3 c$, which should give rise to a radio peak on timescales of a few years (Nakar \& Piran 2011, 2018; Alexander et al. 2017). Finally, the full radio light curve of GW170817 will be crucial for calorimetry, as it will capture all of the energy in the ejecta. The total energy will further shed light into whether GW170817 is a standard short GRB viewed off-axis or it represents a distinct phenomenon.

\section{Conclusion}

We have presented new ATCA and VLA observations of GW170817 covering the period 125-200 days post-merger. Combined with previous radio observations these data show no evidence for spectral evolution, but they conclusively show that the radio counterpart has peaked in brightness at $149 \pm 2$ days 
post-merger and is currently declining. We use this to rule out emission being caused by highly energetic, quasi-isotropic outflow or highly energetic, highly relativistic outflow but are not able to uniquely determine the geometry and structure of the actual outflow material. Continued radio monitoring will allow the temporal decay index to be accurately determined, although this may not be sufficient to establish the presence of a successful jet (Nakar \& Piran 2018) and degeneracies in the ejecta total energy and the density of the circum-merger environment may preclude confirmation of any particular model. Polarization measurements and VLBI observations should be able to break this degeneracy and thus distinguish between the models (also see Gill \& Granot 2018).

We thank P. Chang for helpful discussions. T.M. acknowledges the support of the Australian Research Council through grant FT150100099. A.C. acknowledges support from the NSF CAREER award \#1455090. Part of this research was conducted by the Australian Research Council Centre of Excellence for All-sky Astrophysics (CAASTRO), through project number CE110001020. Part of this work was supported by the Global Relay of Observatories Watching Transients Happen (GROWTH) project funded by the National Science Foundation under PIRE grant No. 1545949. D.K. was additionally supported by by NSF grant AST-1412421. The National Radio Astronomy Observatory is a facility of the National Science Foundation operated under cooperative agreement by Associated Universities, Inc. K.P.M. is currently a Jansky Fellow of the National Radio Astronomy Observatory. The Australia Telescope is funded by the Commonwealth of Australia for operation as a National Facility managed by CSIRO.

Facilities: ATCA, VLA.

Software: Astropy (The Astropy Collaboration et al. 2018), MIRIAD (Sault et al. 1995), DIFMAP (Shepherd 1997), CASA (McMullin et al. 2007).

\section{ORCID iDs}

Dougal Dobie (i) https://orcid.org/0000-0003-0699-7019

David L. Kaplan (iD https://orcid.org/0000-0001-6295-2881

Tara Murphy (10) https://orcid.org/0000-0002-2686-438X

Emil Lenc (1) https://orcid.org/0000-0002-9994-1593

Kunal P. Mooley (i) https://orcid.org/0000-0002-2557-5180

Christene Lynch (i) https://orcid.org/0000-0002-0494-192X

Alessandra Corsi (iD https://orcid.org/0000-0001-8104-3536

Mansi Kasliwal (iD https://orcid.org/0000-0002-5619-4938

\section{References}

Abbott, B. P., Abbott, R., Abbott, T. D., et al. 2017a, PhRvL, 119, 161101 Abbott, B. P., Abbott, R., Abbott, T. D., et al. 2017b, ApJL, 848, L13
Abbott, B. P., Abbott, R., Abbott, T. D., et al. 2017c, ApJL, 848, L12 Adams, S. M., Kasliwal, M. M., Blagorodnova, N., et al. 2017, GCN, 21816, 1 Alexander, K. D., Berger, E., Fong, W., et al. 2017, ApJL, 848, L21 Arcavi, I., Hosseinzadeh, G., Howell, D. A., et al. 2017, Natur, 551, 64 Berger, E., Kulkarni, S. R., \& Frail, D. A. 2004, ApJ, 612, 966 Coulter, D. A., Foley, R. J., Kilpatrick, C. D., et al. 2017, Sci, 358, 1556 Cowperthwaite, P. S., Berger, E., Villar, V. A., et al. 2017, ApJL, 848, L17 D’Avanzo, P., Campana, S., Ghisellini, G., et al. 2018, arXiv:1801.06164 Drout, M. R., Piro, A. L., Shappee, B. J., et al. 2017, Sci, 358, 1570 Evans, P. A., Cenko, S. B., Kennea, J. A., et al. 2017, Sci, 358, 1565 Fong, W., Berger, E., Margutti, R., \& Zauderer, B. A. 2015, ApJ, 815, 102 Frail, D. A., Waxman, E., \& Kulkarni, S. R. 2000, ApJ, 537, 191 Gill, R., \& Granot, J. 2018, MNRAS, submitted (arXiv:1803.05892)

Goldstein, A., Veres, P., Burns, E., et al. 2017, ApJL, 848, L14 Gottlieb, O., Nakar, E., \& Piran, T. 2018, MNRAS, 473, 576

Gottlieb, O., Nakar, E., Piran, T., \& Hotokezaka, K. 2017, arXiv:1710.05896 Granot, J., Panaitescu, A., Kumar, P., \& Woosley, S. E. 2002, ApJL, 570, L61

Haggard, D., Nynka, M., Ruan, J. J., et al. 2017, ApJL, 848, L25

Haggard, D., Nynka, M., Ruan, J. J., Evans, P., \& Kalogera, V. 2018, ATel, 11242,1

Hallinan, G., Corsi, A., Mooley, K. P., et al. 2017, Sci, 358, 1579

Hotokezaka, K., Kiuchi, K., Shibata, M., Nakar, E., \& Piran, T. 2018, arXiv:1803.00599

Kasliwal, M. M., Nakar, E., Singer, L. P., et al. 2017, Sci, 358, 1559

Lazzati, D., Perna, R., Morsony, B. J., et al. 2017, arXiv:1712.03237

Levan, A. J., Lyman, J. D., Tanvirand, I., Mandel, N. R., et al. 2017, GCN, 22207, 1

Lipunov, V. M., Gorbovskoy, E., Kornilov, V. G., et al. 2017, ApJL, 850, L1

Lyman, J. D., Lamb, G. P., Levan, A. J., et al. 2018, arXiv:1801.02669

Margutti, R., Alexander, K. D., Xie, X., et al. 2018, arXiv:1801.03531

Margutti, R., Berger, E., Fong, W., et al. 2017, ApJL, 848, L20

McMullin, J. P., Waters, B., Schiebel, D., Young, W., \& Golap, K. 2007, in ASP Conf. Ser. 376, Astronomical Data Analysis Software and Systems XVI, ed. R. A. Shaw, F. Hill, \& D. J. Bell (San Francisco, CA: ASP), 127

Mooley, K. P., Nakar, E., Hotokezaka, K., et al. 2018, Natur, 554, 207

Mooley, K. P., \& Mooley, S. 2017, GCN, 22211, 1

Nakar, E., \& Piran, T. 2011, Natur, 478, 82

Nakar, E., \& Piran, T. 2018, arXiv:1801.09712

Nakar, E., Piran, T., \& Granot, J. 2002, ApJ, 579, 699

Piro, A. L., \& Kollmeier, J. A. 2018, ApJ, 855, 103

Resmi, L., Schulze, S., Ishwara Chandra, C. H., et al. 2018, ApJ, submitted (arXiv: 1803.02768)

Rossi, E. M., Lazzati, D., Salmonson, J. D., \& Ghisellini, G. 2004, MNRAS, 354,86

Ruan, J. J., Nynka, M., Haggard, D., Kalogera, V., \& Evans, P. 2018, ApJL, 853, L4

Sault, R. J., Teuben, P. J., \& Wright, M. C. H. 1995, in ASP Conf. Ser. 77, Astronomical Data Analysis Software and Systems IV, ed. R. A. Shaw, H. E. Payne, \& J. J. E. Hayes (San Francisco, CA: ASP), 433

Shepherd, M. C. 1997, in ASP Conf. Ser. 125, Astronomical Data Analysis Software and Systems VI, ed. G. Hunt \& H. Payne (San Francisco, CA: ASP), 77

Soares-Santos, M., Holz, D. E., Annis, J., et al. 2017, ApJL, 848, L16

Tanvir, N. R., Levan, A. J., González-Fernández, C., et al. 2017, ApJL, 848, L27

The Astropy Collaboration, Price-Whelan, A. M., Sipőcz, B. M., et al. 2018, arXiv: 1801.02634

Troja, E., \& Piro, L. 2018, ATel, 11245, 1

Troja, E., Piro, L., Ryan, G., et al. 2018, arXiv:1801.06516

Troja, E., Piro, L., van Eerten, H., et al. 2017, Natur, 551, 71

Valenti, S., Sand, D. J., Yang, S., et al. 2017, ApJL, 848, L24 